\title{
Light it up
}

\section{By Tracey Baas, Senior Editor}

Poor tumor definition is a central challenge in cancer surgery, which aims to remove all malignant cells while preserving normal tissue. Now, a team from The University of Texas Southwestern Medical Center has designed fluorescent nanoparticles that can light up tumors regardless of genotype or phenotype. ${ }^{1}$ The approach could allow surgeons to achieve more complete tumor resection.

The new nanoparticle technology is designed to enable real-time imaging in the operating room and provide surgeons with a better means of identifying cancerous tissues than other fluorescent imaging reagents.

Many imaging agents rely on cancer cell-specific biomarkers such as HER2 (EGFR2; ErbB2; neu), epidermal growth factor receptor (EGFR)

"We think the strength of our nanoparticle system is the ultrasensitive $\mathrm{pH}$ response that arises from noncovalent self-assembly of the nanoprobes."

- Jinming Gao,

The University of Texas Southwestern Medical Center and folate receptors. ${ }^{2-4}$ But these targets are expressed in only small subsets of patients, and as a result targeted imaging reagents cannot be applied in a wide range of cancers.

In addition, the fluorescent antibody conjugates that bind to these biomarkers take a long time to clear from the body, and their use is limited by the high background fluorescence in the blood caused by the conjugate being perpetually in an active mode.

Instead of relying on tumor-associated protein targets and a continuously 'on' imaging agent, the Texas team used nanoparticles that could switch on as needed and exploited the slight but consistent $\mathrm{pH}$ differences between normal tissue and the tumor microenvironment.

\section{Let there be light}

To create the on/off nanoparticles, the team developed ultra $\mathrm{pH}$ sensitive (UPS) nanoprobes that differentiated between the relatively close $\mathrm{pH}$ values found in blood and in the extracellular microenvironment of tumor cells (see Figure 1, "Ultra pH-sensitive (UPS) nanoprobes shed new light on tumors").

The nanoprobes could distinguish $\mathrm{pH}$ values less than 0.25 units apart, whereas standard small molecule $\mathrm{pH}$ sensors have a sensitivity of $2 \mathrm{pH}$.

The nanoparticles exist as self-assembled micelles at the higher $\mathrm{pH}$ of the blood ( $\mathrm{pH}$ 7.4) and disassemble when they reach the acidic extracellular microenvironment of tumors $(\mathrm{pH} 6.5-6.8)$ or if they are internalized into acidic endocytic organelles ( $\mathrm{pH} 5.0-6.0){ }^{5}$

The $\mathrm{pH}$-sensitive switch is provided by a fluorophore that stays off because of self-quenching when it is densely packed within the micellar nanoparticles. When the particles dissociate at a lower $\mathrm{pH}$, the fluorescent groups are exposed and the nanoprobes activate.

To target the extracellular tumor microenvironment, the team synthesized UPS nanoprobes based on polyethylene glycol (PEG) that dissociated at $\mathrm{pH}$ 6.9. To target intracellular endosomes and lysosomes, the group synthesized UPS nanoprobes based on PEG-bpoly(2-(diisopropylamino)ethyl methacrylate) (PDPA) that dissociated at $\mathrm{pH} 6.2$.

The nanoprobes contained either red fluorescent tetramethylrhodamine or green fluorescent rhodamine $\mathrm{G}$.

To direct the UPS nanoprobes to the highly angiogenic tumor vasculature, the team modified the nanoparticle surface with peptides containing the arginine-glycine-aspartate (RGD) motif. RGD binds to the angiogenic protein integrin $\alpha_{v} \beta_{3}$ (CD51/CD61), which leads to internalization of the nanoprobe through endocytosis.

In both cases, the nanoprobes incorporated a near-infrared fluorophore and had about a 100-fold signal increase compared with antibody-conjugate approaches.

In mice with lung cancer xenografts, UPS ${ }_{e}$ and UPS nanoparticles accumulated in tumors, and the fluorescent signal increased 11- and 14-fold, respectively, over 24 hours compared with control.

The UPS and UPS nanoprobes also showed 355- and 628-fold greater signal amplifications in the tumor microenvironment than in normal blood.

One potential safety concern was that sporadic spots of UPS signal were seen outside the tumor vasculature-biodistribution studies showed that the nanoprobe was also picked up by the liver and spleen. However, liver and kidney function were not significantly altered.

Similar imaging results were obtained in 10 different mouse models of cancer with diverse tumor phenotypes and organ sites.

Results were published in Nature Materials.

\section{Light at the end of the clinic}

Since publishing the study, the Texas team has adapted the technology to incorporate indocyanine green, an FDA-approved near-infrared dye.

"We believe our technology can help surgeons visualize tumor margins better, as well as sentinel nodes, to achieve complete resection and prevent tumor recurrence," said Jinming Gao, a professor of oncology and pharmacology at the UT Southwestern Medical Center. "We will focus on head and neck, breast and skin cancers as our entry point. We are looking to team up with a company for GMP scale up and preclinical toxicology studies."

Gao said that the team's next step is to "translate the technology into the operating room setting with a clinical camera such as the SPY Elite system for fluorescence-guided surgical resection of solid tumors."

SPY Elite is marketed by the LifeCell Corp. unit of Kinetic Concepts Inc.

"We think the strength of our nanoparticle system is the ultrasensitive $\mathrm{pH}$ response that arises from noncovalent self-assembly of the nanoprobes driven by the fine-tuning of the hydrophobicity of the polymer segments," said Gao.

According to Gao, many existing systems require breaking covalent 


\section{ANALYSIS}

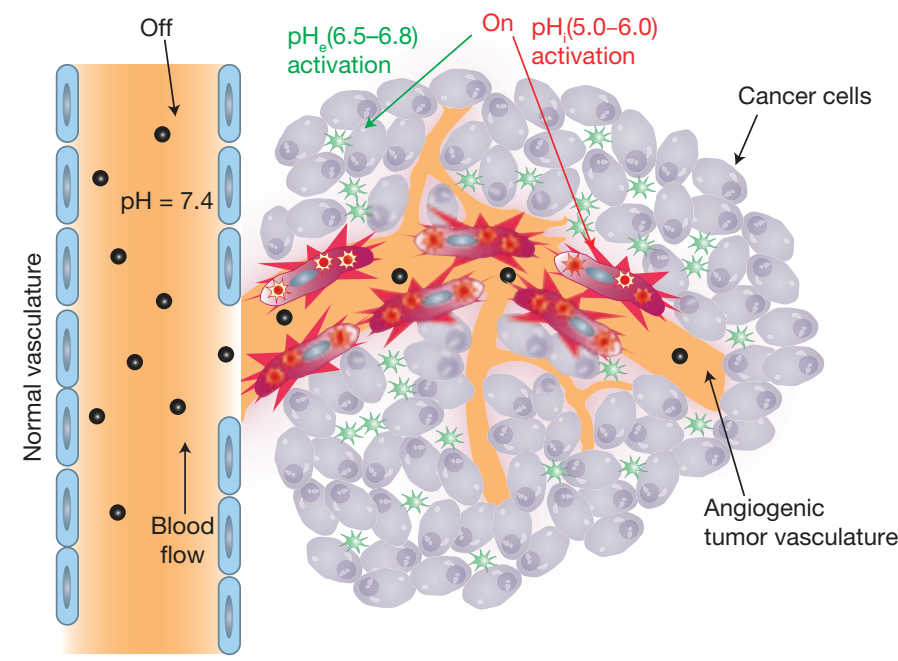

Figure 1. Ultra pH-sensitive (UPS) nanoprobes shed new light on tumors. The UPS nanoprobes stay 'off' at pH 7.4 in normal blood vasculature. After reaching tumors, the UPS nanoprobes are turned 'on' when the nanoparticles disassemble in the acidic extracellular $\mathrm{pH}\left(\mathrm{pH}_{\mathrm{e}} 6.5-6.8\right)$ within the tumor environment or disassemble in the intracellular $\mathrm{pH}\left(\mathrm{pH}_{\mathrm{i}} 5.0-6.0\right)$ of endocytic organelles within the endothelial cells of angiogenic tumor vasculature. (Figure based on Figure 1 in ref. 1.)

bonds to achieve $\mathrm{pH}$ sensitivity, which increases response time because the energy barrier is higher than that in systems that use a noncovalent strategy.

Gooitzen van Dam said that the tunable on/off switch was a breakthrough. van Dam is a professor of surgery and head of the Intraoperative Imaging Research Group at the University of Groningen. He performed the first fluorescent-guided ovarian cancer surgery in September 2011 using a fluorescent folate conjugate.

"The new strategy will be an extremely valuable adjunct to the field of intraoperative imaging, although I have some concerns in relation to the margins of the tumor to be visualized. Usually tumor margins tend to be not extremely hypoxic and thus not extremely acidic," said van Dam.

He also wanted to know more about why some nanoprobes made their way to the liver and the spleen.

Philip Low said that head-to-head comparisons of Gao's fluorescent nanoparticle with other tumor-specific fluorescent imaging agents would be informative.

Low is a professor of chemistry at Purdue University and invented the folate-based imaging technology that van Dam used in his 2011 surgery.

"I suspect Gao's method may have more applicability to a broad range of cancers than most other tumor-imaging methods," Low said.

He cautioned that the tool may have limited applicability in metastases because both tumor accumulation and induction of nanoparticle fluorescence rely on the increased vascular permeability and reduced $\mathrm{pH}$ of the tumor. Those conditions, he said, may not occur in all cancer tissues-especially small clusters of malignant cells.

UT Southwestern Medical Center has filed for a patent covering its imaging technology that will spin out into a startup company.

The fluorescent nanoprobes would also compete with nonfluorescent techniques for aiding tumor resection including the MarginProbe System from Dune Medical Devices Ltd. MarginProbe is a radiofrequencybased tool used during surgery on excised tissue that creates an electromagnetic signature to help define tumor borders. MarginProbe is approved in the U.S. and Europe.

Blaze Bioscience Inc. is running Phase I trials of its BLZ-100 (formerly CyTP 007) to visualize skin cancer. The product is a combination of a fluorescent dye that emits near-infrared light and a targeting peptide that binds to and is internalized by cancer cells.

Baas, T. SciBX 7(1); doi:10.1038/scibx.2014.1

Published online Jan. 9, 2014

\section{REFERENCES}

1. Wang, Y. et al. Nat. Mater.; published online Dec. 8, 2013; doi:10.1038/nmat3819

Contact: Jinming Gao, The University of Texas Southwestern Medical Center, Dallas, Texas

e-mail: jinming.gao@utsouthwestern.edu

2. Urano, Y. et al. Nat. Med. 15, 104-109 (2009)

3. van Dam, G.M. et al. Nat. Med. 17, 1315-1319 (2011)

4. Ke, S. et al. Cancer Res. 63, 7870-7875 (2003)

5. Zhou, K. et al. Angew. Chem. Int. Ed. 50, 6109-6114 (2011)

\section{COMPANIES AND INSTITUTIONS MENTIONED}

Blaze Bioscience Inc., Seattle, Wash.

Dune Medical Devices Ltd., Caesarea Industrial Park, Israel

Kinetic Concepts Inc. (NYSE:KCl), San Antonio, Texas

LifeCell Corp. (NASDAQ:LIFC), Branchburg, N.J.

Purdue University, West Lafayette, Ind.

University of Groningen, Groningen, the Netherlands The University of Texas Southwestern Medical Center, Dallas, Texas 